\title{
Yaws in Pygmy and Bantu children inhabiting the rural zones of Central Africa
}

\author{
Emilia Bylicka-Szczepanowska', Krzysztof Korzeniewski²,3, Dagmara Pokorna-Kałwak \\ ${ }^{1} 4^{\text {th }}$ Department of Infectious Diseases, Provincial Hospital for Infectious Diseases, Warsaw, Poland \\ 2Department of Epidemiology and Tropical Medicine, Military Institute of Medicine, Warsaw, Poland \\ ${ }^{3}$ Department of Tropical Medicine and Epidemiology, Institute of Maritime and Tropical Medicine of Gdynia, Medical University \\ of Gdansk, Poland \\ ${ }^{4}$ Department of Family Medicine, Wroclaw Medical University, Wroclaw, Poland
}

Adv Dermatol Allergol 2022; XXXIX (5): 887-892

DOI: https://doi.org/10.5114/ada.2021.109604

\begin{abstract}
Introduction: Yaws, an infectious dermatosis is one of 17 neglected tropical diseases still present in the world despite the efforts aiming at the eradication of the disease undertaken in 2020. It is mainly found in populations living in poor hygiene conditions with a limited access to water and health care facilities. BaAka Pygmies and Bantu people inhabiting the rural areas of the Central African Republic (CAR) are still affected by yaws on a large scale. Aim: To assess the prevalence of yaws in Central Africa, treatment accessibility, and the need for implementing future health programs.

Material and methods: Primary school BaAka Pygmy and Bantu children from the Dzanga Sangha region (CAR) were assessed in the context of yaws prevalence in years 2019-2020. The diagnosis was based on the clinical picture of the typical skin lesions and their location. Serologic tests were not performed.

Results: Four hundred and ninety-four Pygmies and 235 Bantu children were examined, of whom $38.7 \%$ and $43.0 \%$, respectively, presented primary yaws lesions. The mean age of Pygmy and Bantu children with lesions was 9.1 and 9.7 years old, respectively. In both ethnic groups boys predominated. The most common location of yaws lesions were lower legs, ankles, knees and feet.

Conclusions: Yaws, like all other neglected tropical diseases, might become a cosmopolitan skin disease transferred from Africa to Europe and North America by tourists travelling to sub-Saharan destinations in Africa and migrants. The knowledge of tropical skin diseases and ability to make a differential diagnosis might become necessary for each dermatologist in the next 10-20 years.
\end{abstract}

Key words: yaws, Treponema pallidum, Pygmies, Bantu, Central Africa.

\section{Introduction}

Yaws is an infectious dermatosis (endemic treponematose); a neglected tropical disease caused by bacteria Treponema pallidum subspecies pertenue. This bacterium is closely related to Treponema pallidum subsp. pallidum which causes syphilis $(0.02 \%$ of the difference in genome) [1] and two other non-venereal endemic treponematoses: bejel and pinta caused by Treponema pallidum subsp. endemicum and Treponema pallidum subsp. carateum, respectively. The disease is widespread in the tropical humid climate zone, mostly in Africa (Figure 1), Asia, Latin America and West Pacific.
The disease affects the skin, bones and cartilages and is mostly seen in children under 15 years old in populations living in poor hygiene conditions [2]. Humans are considered the only reservoir of the disease with the direct human-to-human transmission. The important factor promoting transmission of the infection is close physical contact, typical for native populations living in small huts, such as BaAka Pygmies inhabiting rural areas of the Dzanga Sangha region. Skin lesions are more often found in boys, on the lower parts of the limbs, which are more prone to minor injuries and which promote the penetration of bacterium when in contact with the infectious secretion from skin lesions from infected individuals [3].

Address for correspondence: Prof. Krzysztof Korzeniewski, Department of Epidemiology and Tropical Medicine, Military Institute of Medicine, Department of Tropical Medicine and Epidemiology, Institute of Maritime and Tropical Medicine, Medical University of Gdansk, Gdynia, Poland, phone: +48 665707 396, e-mail: kkorzeniewski@wim.mil.pl

Received: 11.07.2021, accepted: 8.08.2021. 


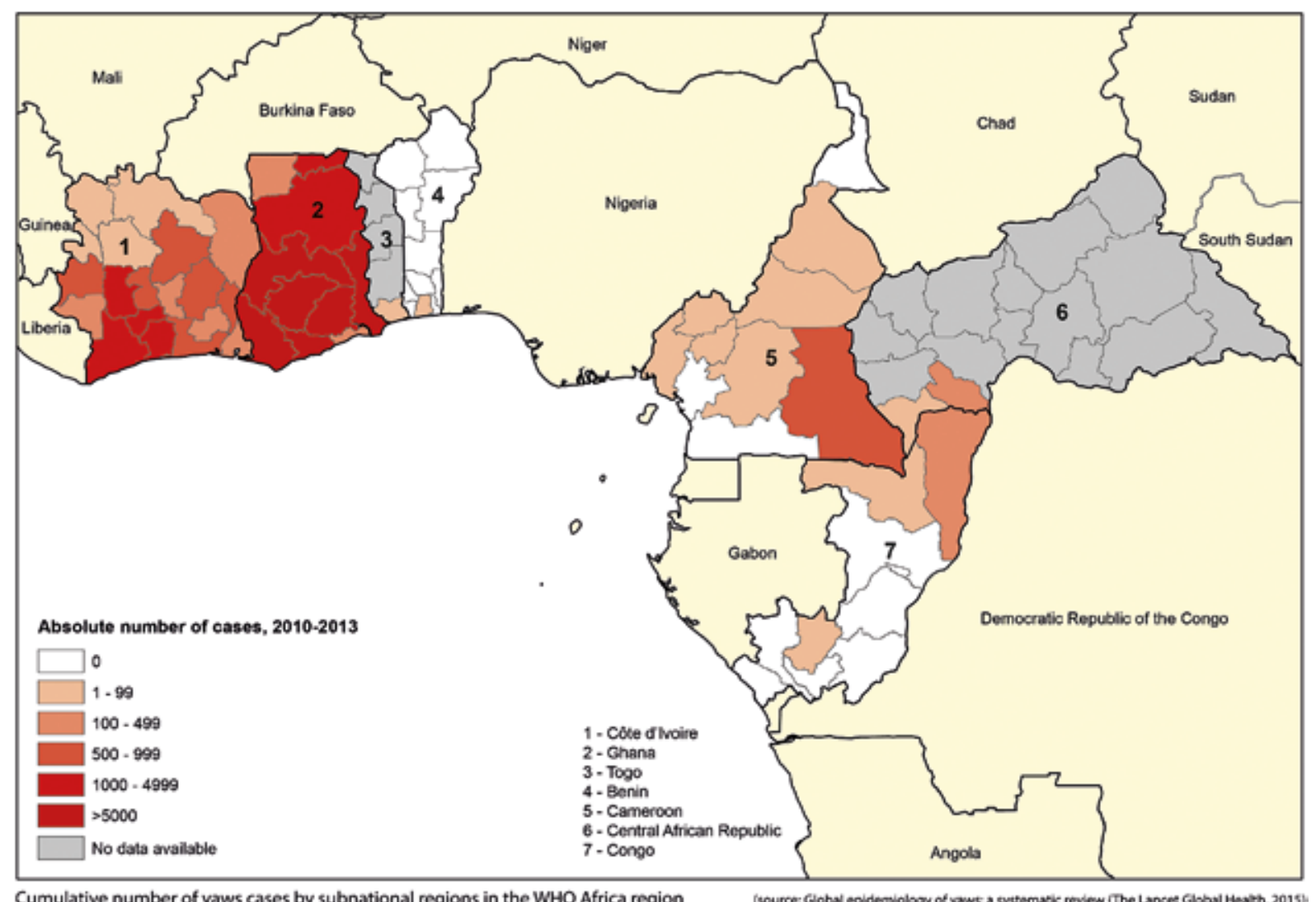

Figure 1. Number of yaws cases in Africa. Source: World Health Organization, available at: https://www.who.int/yaws/ epidemiology, accessed: 13 April 2021

The incubation period takes 9 to 90 days (usually about 21 days) [2]. There are two main stages of the disease - an early stage, when the patient is infective to others, and a late, non-infective stage, manifesting with chronic bone and cartilage deformations disabling the patient's life. In the early stage affecting the skin, yaws manifests by papillomatous lesions forming the crust, called "mother yaw" which evolves within 2-4 weeks into an ulcer with a highly infective mucous secretion. Untreated, it resolves after up to 6 months spontaneously. The treponemes may spread through the bloodstream and in the secondary stage it might reappear all over the body as disseminated smaller papules called "daughter yaws" [1, 4]. At this stage the treatment is highly effective with a single dose of benzathine benzylpenicillin or azithromycin [2, 4]. About $10 \%$ of the untreated patients develop the late, tertiary stage after many years of the latent asymptomatic infection, which manifests by deformations of the bones, nose, cartilages and painful palmoplantar hyperkeratosis.

In endemic regions with a limited access to laboratory facilities with well-trained technicians, the differential diagnosis is mostly based on the clinical manifestation and the medical interview. In such regions, the serologic tests confirm the diagnosis, but will not differentiate yaws from syphilis. Two serologic tests are the most common: nontreponemal rapid plasma reagin (RPR) test and treponemal Treponema pallidum particle agglutination assay (TPPA) detecting antibodies against the bacterium. Both tests are simple and feasible in the laboratories with personnel or experience shortages [5]. The gold standard for yaws diagnostics is polymerase chain reaction ( $P C R$ ) method detecting the DNA of the bacterium Treponema pallidum subsp. Because of the poor availability and high costs of the PCR method, it is used only in research studies [1].

The prevention of yaws is based on the interruption of the human-to-human transmission: education, better hygiene, better access to clean water, regular mass treatment campaigns with benzathine benzylpenicillin in the past and nowadays with azithromycin as well as treatment of contacts. In the eradication approach, called the Morges strategy 2012, the emphasis is on implementing the total community treatment (TCT) strategy, which involves azithromycin distribution to $90 \%$ of the local population [2] with a minimum of two or three rounds of drug distribution in the affected areas, and the total targeted treatment (TTT) strategy consisting in the treatment of 
sick individuals and their contacts. Both strategies should be included in the effective eradication programme [6].

\section{Aim}

The study aims to assess the prevalence of yaws among BaAka Pygmy and Bantu children in Central Africa, treatment distribution, and the need of implementing future health programs.

\section{Material and methods}

Primary school BaAka Pygmy and Bantu children aged 8-10 years living in the rural Dzanga Sangha region (in the south-western parts of the Central African Republic) were assessed in the context of yaws prevalence. The study was carried out in June-October 2019. The diagnosis was based on the clinical picture of the typical skin lesions; children were examined at five different schools attended by both the BaAka Pygmies (a nomadic tribe) and Bantu children (sedentary tribes); their ethnic group, sex, age, the number and location of yaws lesions were taken. Serologic tests to confirm the diagnosis were not performed. Children with yaws-like lesions were given a single dose of azithromycin ( $30 \mathrm{mg} / \mathrm{kg}$ bw), which is the standard treatment regimen recommended by the WHO. Children who did not manifest yaws-specific lesions were entered into the register for statistical purposes.

\section{Ethics approval and consent to participate in the study}

The research task entitled The analysis of health issues affecting Pygmy tribes inhabiting the forests of the Central African Republic was approved by the Bioethics Committee at the Military Institute of Medicine (Resolution No. 137/WIM/2018).
The treatment was administered by consent of the Head of the Education Sector for the Dzanga Sangha region.

\section{Statistical analysis}

All statistical calculations were performed using the statistical package STATA 2019 (data analysis software system) version 16.0. and an Excel spreadsheet. The qualitative variables were represented as counts and percentages. Chi-square $\left(\chi^{2}\right)$ tests of independence were used for qualitative variables and the distributions of quantitative variables were compared using Mann-Whitney test. In all calculations, the level of significance was set at $p=0.05$.

\section{Results}

The study group involved 729 children, including 494 BaAka Pygmies and 235 Bantu. Their skin was examined for any yaws-specific lesions. A total of 191 Pygmies (38.7\%) and 101 Bantu (43.0\%) children presented characteristic yaws lesions. There was no statistically significant difference in the prevalence of yaws lesions between Pygmy and Bantu children. In the group involving Pygmy children (304 males, 190 females) skin lesions were more often found in boys than in girls (116 males vs. 75 females). A similar finding was observed in the group of Bantu children (127 males vs. 108 females) where boys with yaws lesions predominated over girls (58 males vs. 43 females). The mean age of all Pygmy and Bantu children participating in the study was 8.6 years and 9.1 years, respectively. The mean age of Pygmy children with yaws-specific lesions was 9.1 years and of those without skin lesions -8.2 years, whereas the mean age of Bantu children with yaws lesions was 9.7 years, and those without skin lesions -8.6 years. Skin lesions mainly affected the lower limbs (Figures 2-4), in particular the lower leg, the bridge of the foot, the heel and the knee (Table 1).
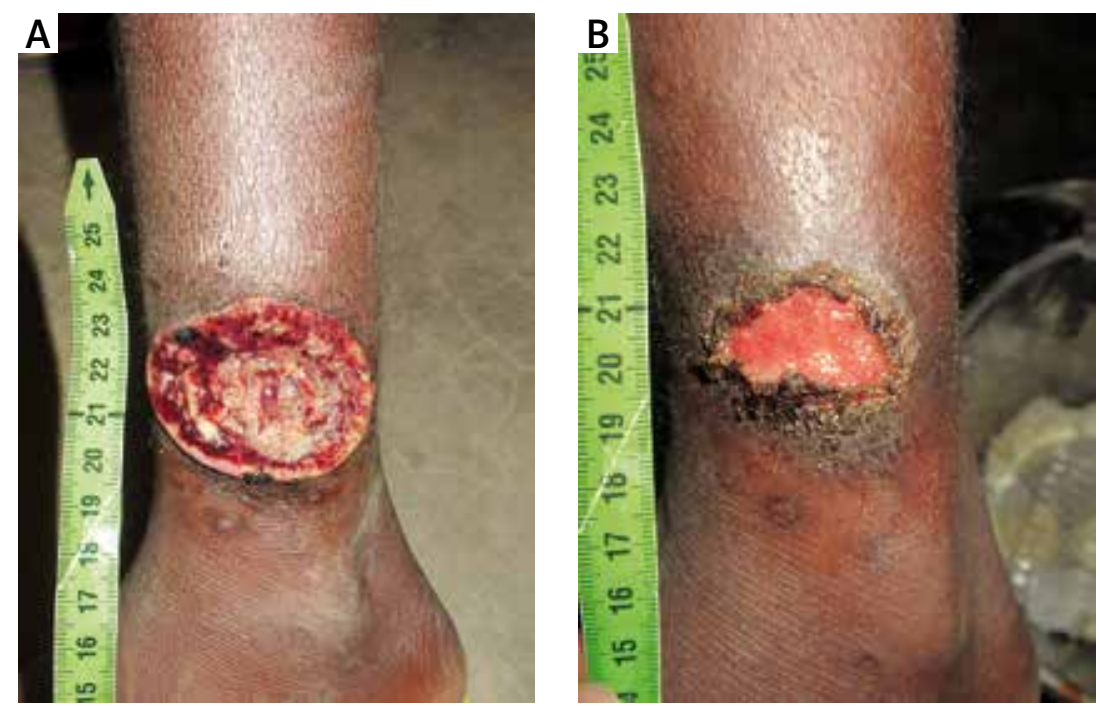

Figure 2. Yaws manifested by an ulcer on the leg, 9-year-old Pygmy boy (A) and 20 days after a single dose of treatment with 1.2 million IU of benzathine benzylpenicillin (B) 


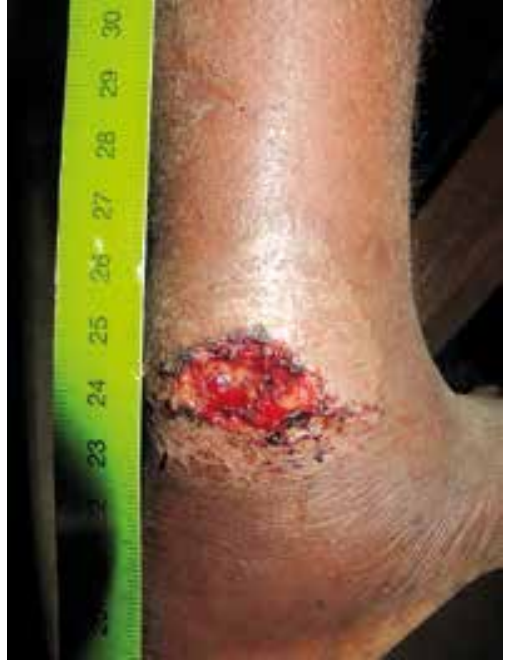

Figure 3. Yaws manifested by an ulcer on the leg, 8-yearPygmy boy; treatment: single dose of benzathine benzylpenicillin 600,000 IU

Table 1. Location of skin lesions in the study sample $(n=729)$

\begin{tabular}{lccc}
\hline Location of skin lesions & Total $(n)$ & Pygmies $(n)$ & Bantu $(n)$ \\
\hline Foot bridge & 60 & 33 & 27 \\
\hline Heel & 9 & 5 & 4 \\
\hline Ankle & 73 & 50 & 23 \\
\hline Lower leg & 100 & 61 & 39 \\
\hline Knee & 57 & 41 & 16 \\
\hline Thigh & 7 & 6 & 1 \\
\hline Buttock & 1 & 1 & 0 \\
\hline Elbow & 2 & 2 & 0 \\
\hline Arm & 2 & 1 & 1 \\
\hline Head & 1 & 0 & 1 \\
\hline
\end{tabular}

Unilateral yaws lesions were more often seen in Pygmies (86.9\% vs. Bantu 79.2\%); whereas bilateral lesions were more common in Bantu (20.8\% vs. Pygmies 13.1\%); Bantu children presented with more lesions compared to Pygmies (Table 2).

\section{Discussion}

The World Health Organization launched a campaign to eradicate yaws as early as in the 1950s [3] when mass treatment campaigns of patients and their contacts with benzathine benzylpenicillin - the only antibiotic effective against yaws at that time - were initiated. Seventy years ago yaws was considered a major public health issue potentially affecting as many as 50 million people in Africa, South-East Asia, West Pacific and Latin America $[2,7]$. The initial success of the programme has led to

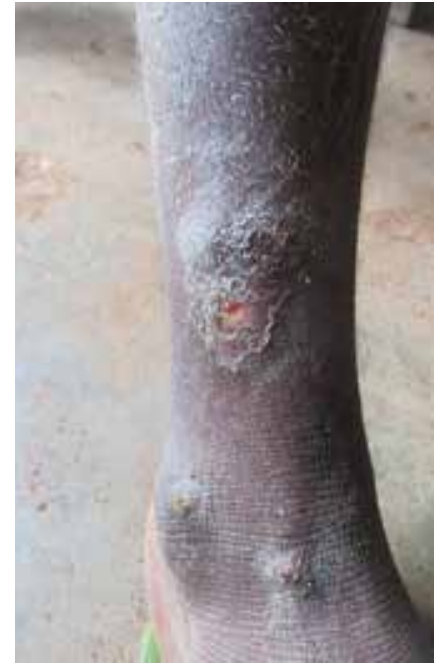

Figure 4. Multiple yaws lesions on the leg, 10-year-old Bantu boy; treatment: single dose of azithromycin $900 \mathrm{mg}$

Table 2 . The number of skin lesions in the study sample $(n=729)$

\begin{tabular}{lcc}
\hline Parameter & BaAka & Bantu \\
\hline Number of lesions & 1.4 & 2.0 \\
\hline$P$-value (Mann-Whitney) & \multicolumn{3}{c}{0.01} \\
\hline
\end{tabular}

a significant reduction in the number of new yaws cases [7] with $95 \%$ of the infected patients having been cured [8]. However, the global efforts to eradicate the disease have failed and nowadays yaws is still being diagnosed in many parts of the world [2]. Currently, yaws is known to be endemic in 15 different countries across the world, including in the Central African Republic (CAR) [2]. The WHO report of 2018 assumed that the disease would be eradicated by 2020 [9], but clearly the eradication goal has not been achieved. The eradication efforts are hampered by poverty and poor hygiene conditions which are a major problem in the so-called 'failed states', such as the CAR. The Central African Republic is known to be one the poorest countries in the world. In fact, its GDP (gross domestic product) per capita was USD 467.9 in 2019, which makes it the fifth poorest country globally [10].

With a correct diagnosis, the cure rate of yaws is $100 \%$. Until now, there has been no reported resistance to benzathine benzylpenicillin. A single dose of the antibiotic administered intramuscularly (dosing should be adjusted to body weight) is enough to fight the infection [11]. A study published in 2012 demonstrated that azithromycin was as effective in treating yaws as penicillin $[12,13]$. Consequently, mass treatment of yaws with azithromycin was soon initiated. The use of azithromycin in the treatment of yaws not only simplified mass treatment campaigns but also lowered the costs. The current 
WHO target is to eradicate yaws by $2030[14,15]$, which is an optimistic scenario considering the fact that in some cases skin lesions fail to resolve despite recommended treatment (although as yet there has been no confirmed resistance of T. pallidum subsp. pertenue to penicillin and azithromycin resistance rates are low) [16]. This may be due to the fact that some yaws-like lesions may have a more complex aetiology, and resistance to treatment can be explained by the presence of other etiological factors, including Haemophilus ducreyi [2], Streptococcus pyogenes [17] as well as pathogens responsible for cutaneous leishmaniosis [18], leprosy [18] or Buruli ulcer $[18,19]$.

Our study found that a considerable proportion of children inhabiting the Dzanga Sangha region of the Central African Republic, both BaAka Pygmy and Bantu, present with yaws-specific skin lesions. Surprisingly, yaws rates were higher in Bantu children although they are more likely to live in better hygiene conditions than the nomadic Pygmies [20]. Several studies are available in the literature on the prevalence of yaws in the CAR. A study by Widy-Wirski et al. which was carried out in the late 1970s found yaws lesions in $50 \%$ of the BaAka Pygmies involved in the study. Whereas, serologic tests confirmed the presence of T. pallidum in $86 \%$ of children and $95 \%$ of adults [21]. The same study also assessed yaws prevalence in Bantu people. Symptomatic infections were found in $30 \%$ of Bantu participants, but serologic tests revealed the infection in as many as 78\% of children and $98 \%$ of adults. Saradon et at. and Martin et al. carried out similar studies into the prevalence of yaws in Pigmies inhabiting the Sangha region and found that yaws cases were rare among the participants [22]. Such a considerable reduction in the number of cases was possible because of the implementation of total mass treatment (TMT) in 1980 and surveillance of yaws infections between 1980 and 1985 [22]. Another study, carried out in 2008-2009 in the Lobaye prefecture of the CAR (a region neighbouring Congo-Brazzaville and the Democratic Republic of the Congo) found yaws-specific lesions in 10\% of the Pygmies involved in the study. All the Pygmy population participating in the study received appropriate treatment [4]. Studies conducted by the authors of the present article between 2019 and 2020 demonstrated that the prevalence of yaws skin lesions was higher in Bantu children (43.0\%) than in Pygmy children (38.7\%).

One of the study limitations was reducing the study sample to school children only. Since adults were excluded from the study, it was impossible to assess yaws prevalence in the general population. Instead, our study focused on school-age children, who have the highest risk of developing the infection. The results, which are in line with other authors' findings, confirm that yaws most commonly affects boys aged 6-10 years, and that the lesions most commonly affect the lower legs [2]. Another limitation of the research study was the lack of serologic tests which would confirm Treponema pallidum subsp. infection; although in areas where yaws is endemic the diagnosis is normally established on the basis of clinical manifestation of lesions by trained health care providers. The lack of testing is primarily attributable to the limited access to laboratory facilities as well as shortages of qualified personnel and a lack of financial resources.

\section{Conclusions}

The World Health Organization recognizes yaws as a neglected tropical disease; however, since there have recently been no reports on the prevalence of yaws in endemic regions, including in the Central African Republic, it should rather be considered a forgotten illness. The growth in international tourist arrivals, also in SubSaharan Africa, and the rising interest in low-cost travel increase the risk of developing a neglected or a forgotten disease by travellers from developed countries. Correct diagnosis of a tropical dermatosis speeds up the initiation of the most appropriate treatment. A true value of the study is the demonstration that yaws remains a major public health issue in the Central African Republic. Identification of outbreaks, regular screening, treatment and monitoring of cases are necessary for complete eradication of the disease globally. It is essential that studies similar to ours be conducted at the same locations at regular intervals and that they involve the largest possible number of participants, including adults. It is also recommended that serologic tests be performed.

\section{Conflict of interest}

The authors declare no conflict of interest.

\section{References}

1. Ndzomo Ngono JP, Tchatchouang S, Noah Tsanga MV, et al. Ulcerative skin lesions among children in Cameroon: It is not always Yaws. PLoS Negl Trop Dis 2021; 15: e0009180.

2. World Health Organization. Yaws. Available at: https://www. who.int/news-room/fact-sheets/detail/yaws. Accessed: 6 January 2021.

3. Hackett CJ, Guthe T. Some important aspects of yaws eradication. Bull World Health Organ 1956; 15: 869-96.

4. Manirakiza A, Boas SV, Beyam N, et al. Clinical outcome of skin yaws lesions after treatment with benzathine benzylpenicillin in a pygmy population in Lobaye, Central African Republic. BMC Res Notes 2011; 4: 543.

5. Lubinza CKC, Lueert S, Hallmaier-Wacker LK, et al. Serosurvey of Treponema pallidum infection among children with skin ulcers in the Tarangire-Manyara ecosystem, northern Tanzania. BMC Infect Dis 2020; 20: 392.

6. WHO/Department of Control of Neglected Tropical Diseases. Eradication of yaws - the Morges Strategy. Weekly epidemiological record. Available at: https://www.who.int/ yaws/ resources/who_wer8720/en/. Accessed: 17 May 2021. 
7. Antal GM, Causse G. The control of endemic treponematoses. Rev Infect Dis 1985; 7 (Suppl 2): S220-6.

8. Asiedu K, Fitzpatrick C, Jannin J. Eradication of yaws: historical efforts and achieving WHO's 2020 target. PLoS Negl Trop Dis 2014; 8: e3016.

9. World Health Organization. Report of a global meeting on yaws eradication surveillance, monitoring and evaluation. Geneva, 29-30 January 2018. Accessed: 14 March 2021.

10. World Bank. Available at: https://data.worldbank.org/indicator/NY.GDP.PCAP.CD? locations=CF. Accessed: 6 January 2021

11. Marks M, Mitjà O, Solomon AW, et al. Yaws. Br Med Bull 2015; 113: 91-100.

12. Mitjà O, Hays R, Ipai A, et al. Single-dose azithromycin versus benzathine benzylpenicillin for treatment of yaws in children in Papua New Guinea: an open-label, non-inferiority, randomised trial. Lancet 2012; 379: 342-7.

13. Mabey D. Oral azithromycin for treatment of yaws. Lancet 2012; 379: 295-7.

14. Holmes A, Tildesley MJ, Solomon AW, et al. Modeling treatment strategies to inform yaws eradication. Emerg Infect Dis 2020; 26: 2685-93.

15. World Health Organization. Ending the neglect to attain the sustainable development goals: a road map for neglected tropical diseases 2021-2030: overview. Available at: https:// apps.who.int/iris/handle/10665/332094. Accessed: 6 June 2021.

16. Mitjà $\mathrm{O}$, Godornes $\mathrm{C}$, Houinei W, et al. Re-emergence of yaws after single mass azithromycin treatment followed by targeted treatment: a longitudinal study. Lancet 2018; 391: 1599-607.

17. Griesenauer B, González-Beiras C, Fortney KR, et al. 2021 Streptococcus pyogenes is associated with idiopathic cutaneous ulcers in children on a yaws-endemic island. mBio 2021; 12: e03162-20.

18. Organisation mondiale de la Santé. Comment reconnaître les maladies tropicales négligées selon les altérations de la peau : guide de formation à l'usage du personnel sanitaire de première ligne. Organisation mondiale de la Santé. Available at: https://apps.who.int/iris/handle/10665/275272. Accessed: 06 June 2021.

19. World Health Organization. Buruli ulcer. Available at: https:// www.who.int/health-topics/buruli-ulcer\#tab=tab_2. Accessed: 12 April 2021.

20. Martin PM, Gonzalez JP, Martin MH, et al. Clinical aspects and usefulness of indirect absorbed immunofluorescence for diagnosis of yaws in Central Africa. J Clin Microbiol 1988; 26: 2432-3.

21. Widy-Wirski R, D'Costa J, Meheus A. Prévalence du pian chez les pygmées en Centrafrique. Ann Soc Belg Med Trop 1980; 60: 61-7.

22. Salomon G. Le pian chez les peuples de la forętéquatoriale du Nord-Congo et du sud dela République Centrafricaine. L'homme et la foret tropicale. 1999. Accessed: 15 March 2021. 